\title{
A novel recombinant immuno-tBid with a furin site effectively suppresses the growth of HER2-positive osteosarcoma cells in vitro
}

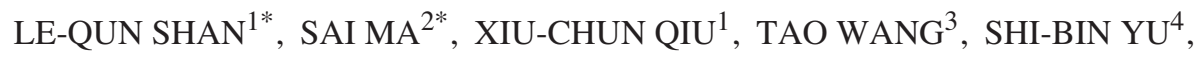 \\ BAO-AN MA ${ }^{1}$, YONG ZHOU ${ }^{1}$, QING-YU FAN ${ }^{1}$ and AN-GANG YANG ${ }^{3}$ \\ ${ }^{1}$ Department of Orthopaedic Surgery, Tangdu hospital, Fourth Military Medical University; \\ ${ }^{2}$ Department of Prosthodontics, School of Stomatology, Fourth Military Medical University; ${ }^{3}$ Department of Immunology, \\ Fourth Military Medical University; ${ }^{4}$ Department of Oral Anatomy and Physiology, School of Stomatology, \\ Fourth Military Medical University, Xi'an 710032, P.R. China
}

Received September 1, 2010; Accepted October 19, 2010

DOI: $10.3892 /$ or.2010.1074

\begin{abstract}
Immunotherapy is a promising strategy for the treatment of human epidermal growth factor receptor 2 (HER2)-positive tumors. Previously, we constructed an immuno-carboxy terminal fragment of Bid (immuno-tBid) and reported its specific and effective destruction of HER2positive tumor cells. In this study, in order to further reduce the immunogenicity of the previous immuno-proapoptotic protein, we constructed a novel immuno-tBid by replacing domain II of Pseudomonas exotoxin A with a short furin cleavage sequence from the diphtheria toxin. In order to explore the possible application of this novel immuno-tBid in the treatment of osteosarcoma, we first examined the expression of the HER2 protein in a subclone of a human osteosarcoma cell line with relatively high metastatic potential (SOSP-9607-E10), as well as in clinical specimens of osteosarcoma. Quantitative real-time PCR and Western blot analysis revealed that the expression of HER2 was upregulated in the SOSP-9607-E10 cells, while immunohistochemical analysis revealed that HER2 was overexpressed in
\end{abstract}

Correspondence to: Professor Qing-Yu Fan, Department of Orthopaedic Surgery, Tangdu hospital, Xi'an 710038, P.R. China E-mail: bonetm@fmmu.edu.cn

Dr An-Gang Yang, Department of Immunology, Fourth Military Medical University, Xi'an 710032, P.R. China

E-mail: agyang@fmmu.edu.cn

*Contributed equally

Abbreviations: HER-2, human epidermal growth factor receptor-2; PEA, Pseudomonas exotoxin A; FBS, fetal bovine serum; EGFR, epidermal growth factor receptor; PBS, phosphate-buffered saline; $\mathrm{AIF}$, apoptosis inducing factor; MAb, monoclonal antibody

Key words: osteosarcoma, gene therapy, human epidermal growth factor receptor-2/neu, Bid, apoptosis
$37 \%$ of the tissue specimens examined. Both HER2-positive SOSP-9607-E10 and SKBR-3 cells, as well as HER2-negative HeLa cells were transiently transfected with the novel immuno-tBid in order to study its specific pro-apoptotic effect. We demonstrate here that this novel immuno-tBid induces the specific destruction of HER2-overexpressing SOSP-9607-E10 cells through the release of cytochrome C. These results suggest that the novel immuno-tBid with a minimized exogenous fragment could represent a competitive approach for the treatment of HER2-positive osteosarcoma.

\section{Introduction}

Immunotoxins, which are hybrid proteins composed of plant or bacterial toxins linked with a targeting molecule, such as a monoclonal antibody (MAb), an antibody fragment, a growth factor, or a cytokine (1-4), have been proven to be effective in destroying tumor cells specifically. Similar to certain other well-demonstrated anti-tumor immunotoxins (5-7), the Pseudomonas exotoxin A (PEA)-based human epidermal growth factor receptor 2 (HER2)-targeted immunotoxin, e23sFv-PEA40, has shown potent and selective cytotoxicity to HER2-positive tumors both in vitro and in vivo (8). However, most of the clinical trials of the immunotoxin encountered the same obstacle, in that the foreign toxin fragments in immunotoxins induced strong humoral immune responses (9-14). In addition to the development of neutralizing antibodies against the plant or bacteria-derived toxins $(9,10)$ which profoundly diminished the anti-tumor efficiency of the therapy, other side-effects including vascular leakage, and non-specific toxicity to vascular endothelial, liver, or renal cells $(7,10-14)$ were also induced by immunotoxins. Therefore, studies are now focusing on immunotoxins containing human-derived effector proteins, such as human pro-apoptotic proteins, in order to minimize non-specific toxicity and immunogenicity (15).

Previously, we reported the potent and specific suppressing effect of the partially humanized fusion protein, immunocarboxy terminal fragment of Bid (immuno-tBID), composed 
of a HER2-specific single-chain antibody, the PEAII domain and tBid, on HER2-overexpressing human breast cancer cells (SK-BR-3) and human ovarian cancer cells (SK-OV-3) $(16,17)$. In order to further reduce the exogenous fragment and thus minimize immunogenicity, we constructed a novel class of immuno-proapoptotic proteins by replacing the PEAII domain of previous immuno-proapoptic proteins by a short furin cleavage sequence from the diphtheria toxin $\left(\mathrm{A}_{187} \mathrm{GNRVRRSVG}_{196}, \mathrm{Fdt}\right.$ ), and confirmed their efficiency in inducing cell death in vitro and reducing tumor size in vivo (18). Even though these novel immuno-proapoptotic proteins have been proven to be effective in inducing apoptosis in the HER2-overexpressing human breast tumor cell line, SKBR-3, and the human gastric cancer cell lines, SGC-7901 and AGS, their effect on human osteosarcoma has not yet been investigated. Thus the purpose of the present study, was to modify our previous immuno-tBid by introducing the short furin cleavage sequence, Fdt, and to study the possible proapoptotic effect of this novel immuno-tBid on HER2-overexpressing SOSP-9607-E10 cells in vitro.

\section{Materials and methods}

Construction of expression plasmids. The gene encoding truncated Bid and the single-chain HER2 antibody (e23sFv), were generated in our previous study (16). The furin site sequence from the diphtheria toxin $\left(\mathrm{A}_{187} \mathrm{GNRVRRSVG}_{196}\right.$, Fdt) was incorporated into tBid by PCR. The novel immuno$t$ Bid gene was generated by the sequential fusion of the genes of a signal peptide (MKHLWFFLLLVAAPRWVLS), e23sFv, the furin cleavage sequence, Fdt, and tBid (bid $\Delta 1-60$ ). This novel immuno-tBid gene was cloned into the expression vector, pCMV. Vector sequence was confirmed by DNA sequencing.

Cell culture and transfection. The HER2-positive human breast tumor cell line, SKBR-3, the HER2-negative human cervical carcinoma cell line, HeLa, and the subclone of a human osteosarcoma cell line with relatively high metastatic potential, SOSP-9607-E10, were maintained in DMEM or RPMI 1640 (Invitrogen) supplemented with 10\% fetal bovine serum (FBS) and $4 \mathrm{mmol} / \mathrm{l} \mathrm{L}$-glutamine. At $24 \mathrm{~h}$ before transfection, cells were seeded in 12 -well plates at a density of $1 \times 10^{5}$ cells per well. Transfection was performed with Lipofectamine 2000 (Invitrogen) according to the standard procedure. For transient expression, cells were harvested $72 \mathrm{~h}$ after transfection. For stable transfection, cells were selected in the medium containing $800 \mu \mathrm{g} / \mathrm{ml} \mathrm{G} 418$ (Invitrogen) for 2 to 3 weeks.

Immunohistochemical analysis. Paraffin-embedded, formalin-fixed resection specimens from patients were used for the immunohistochemical analyses. The study included 48 specimens from patients with osteosarcoma who presented to the Department of Orthopeadic Surgery in Tangdu Hospital from 2003 to 2004. The inclusion criteria were: Primary osteosarcoma without lung metastasis at the time of diagnosis, pre-operative chemotherapy with paclitaxel, with a postoperative follow-up of 24 months. For immunohistochemical staining, paraffin-embedded tissue sections were dewaxed, hydrated, and incubated in $0.3 \%$ methanol- $\mathrm{H}_{2} \mathrm{O}_{2}$ for $20 \mathrm{~min}$ to remove endogenous peroxidase. After the sections were washed with phosphate-buffered saline (PBS) for three times and blocked with normal goat serum, they were incubated overnight at $4^{\circ} \mathrm{C}$ with mouse anti-HER 2 polyclonal antibody (1:200, M0196, Changdao Corp., China). The sections were then incubated with goat anti-mouse IgG as the secondary antibody for $30 \mathrm{~min}$ and then processed with 3,3'-diaminobenzidine staining.

Quantitative real-time PCR (RT-PCR) for HER2 $m R N A$ in SKBR-3, HeLa and SOSP-9607-E10 cells. Transcriptional levels of HER2 in SKBR-3, SOSP-9607-E10 and HeLa cells, were measured by quantitative RT-PCR using the SYBR Premix Ex Taq kit (Takara Bio, Madison, WI, USA). Total RNA was extracted from cultured cells by using TRIzol ${ }^{\circledR}$ Reagent (Invitrogen). cDNA was synthesized using $2 \mu \mathrm{g}$ of total RNA, oligo(dT) $)_{18}$ primer and Superscript RT (from Invitrogen). Quantitative RT-PCR was performed using $1 \mu \mathrm{l}$ of a 1:5 dilution of first-strand cDNA and primers specific to HER 2 and $B$-actin on a Prism 7500 cycler (Applied Biosystems). HER2 was amplified using the forward primer, 5'-TCA CCT ACA ACA CAG ACA CGT TTG-3', and the reverse primer, 5'-ATC CCA CGT CCG TAG AAG GTA-3'. PCR products were electrophoresed on a $1 \%$ agarose gel, stained with ethidium bromide and visualized under UV light. All the experiments were repeated for at least three times and similar results were obtained.

Western blot analysis. Western blot analysis was performed to study the expression of the HER2 protein in SKBR-3, SOSP-9607-E10 and HeLa cells. Cells were washed with icecold PBS, scraped into ice-cold PBS, and thereafter lysed. The protein content was measured by the Bradford assay using bovine serum albumin as the standard. Protein from cell lysates was separated by SDS-PAGE and transferred to polyvinylidene difluoride membranes (Amersham Biosciences). The blotted membranes were then incubated with primary antibody that recognizes HER2 (1:500; NeoMarkers) overnight at $4^{\circ} \mathrm{C}$ in PBS Tween-20. The membranes were then incubated with horseradish peroxidase-conjugated secondary antibody (1:2000; Zhongshan) for $2 \mathrm{~h}$ at room temperature. Western blots were visualized using an enhanced chemiluminescence kit (Pierce).

Cell viability assay. Cell viability of the transiently transfected cells was tested by using the 3-(4,5-dimethylthiazol2-yl)-2,5-diphenyltetrazolium bromide (MTT) assay. Briefly, cells transfected with $e 23 s F v$-Fdt-tBid were cultured in 96well plates for 24 to $96 \mathrm{~h}$, and then incubated with $20 \mu \mathrm{l}$ of $1.5 \mathrm{mg} / \mathrm{ml} \mathrm{MTT}$ for $4 \mathrm{~h}$. After that, the cells were treated with $150 \mu \mathrm{l}$ DMSO. Values at $\mathrm{A}_{490} \mathrm{~nm}$ were determined using the Sunrise microplate reader (Tecan). Each assay was performed in triplicate on at least three independent occasions.

Cell-killing assay. The cell-killing ratio of the transiently transfected cells was determined by cell counting after trypan blue staining. After the cells were detached and re-suspended in DMEM containing 10\% FBS, they were stained with trypan blue. Unstained cells were counted on a blood cell 


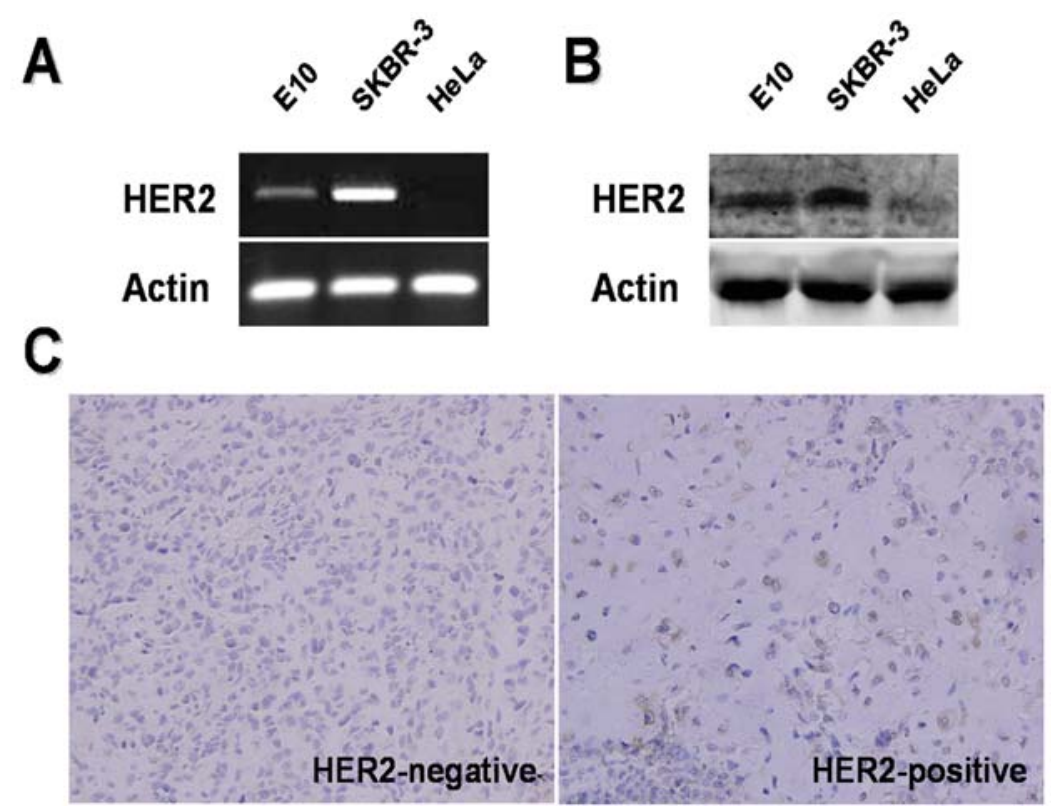

Figure 1. The expression of HER2 in the SOSP-9607-E10 cell line and clinical osteosarcoma specimens. (A) Transcriptional levels of HER2 in SKBR-3, SOSP-9607-E10 and HeLa cells. Overexpression of HER2 was detected in SOSP-9607-E10 cells by RT-PCR. (B) The expression of HER2 protein in SKBR-3, SOSP-9607-E10 and HeLa cells. As can be seen from the results of the Western blot analysis, HER2 was overexpressed in SOSP-9607-E10 cells. (C) Immunohistochemical analysis of the expression of HER2 in clinical osteosarcoma specimens (x400). The negative specimen showed no HER2-positive cells, while the positive specimen revealed numerous HER2-positive osteosarcoma cells.

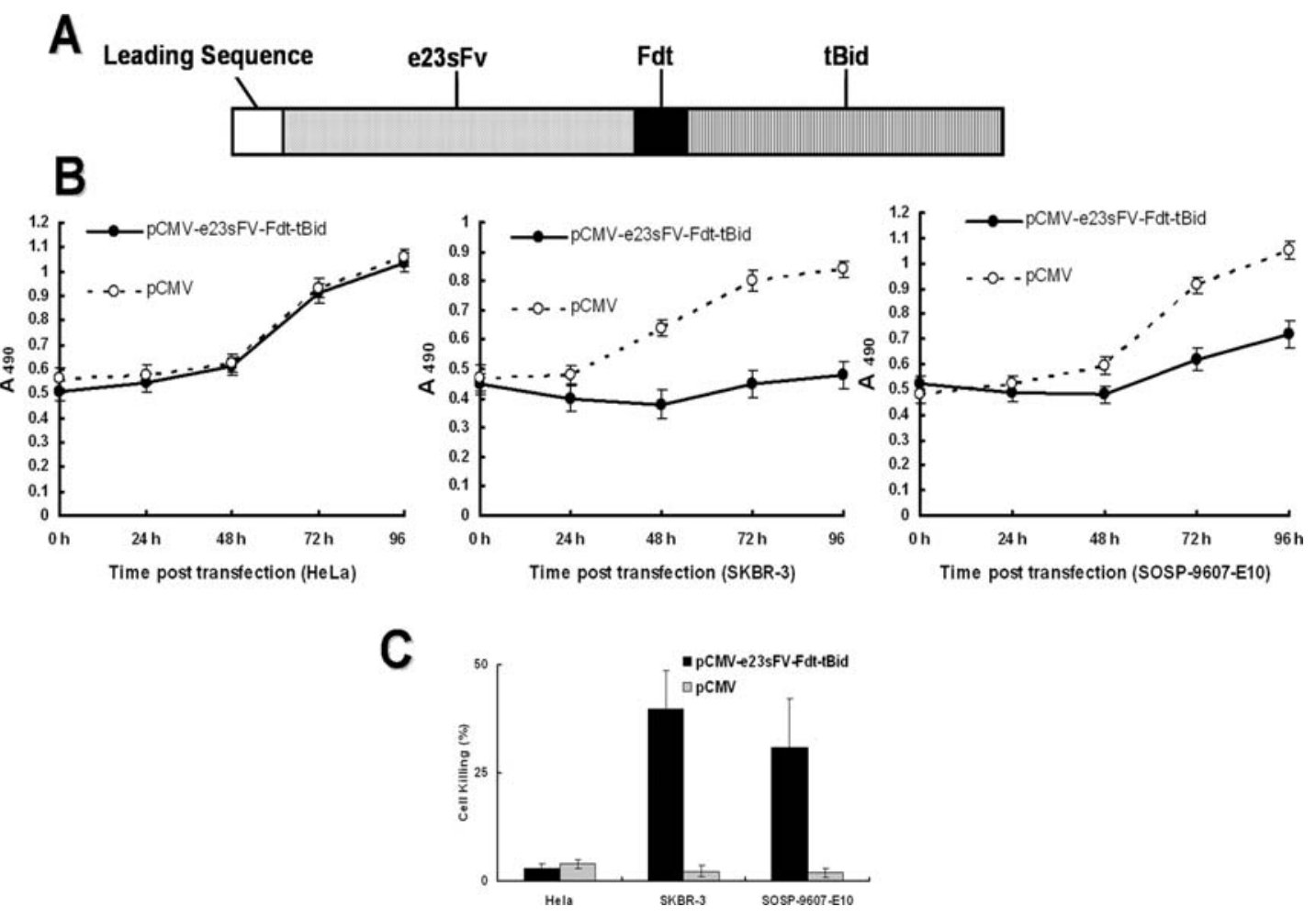

Figure 2. The novel immuno-tBid induced cell death in transiently transfected SOSP-9607-E10 cells. (A) The novel recombinant gene consists of a signal peptide, the HER2 antibody, e23sFv, the furin cleavage sequence, Fdt, and tBID (bid $11-60$ ). (B) MTT assay of SKBR-3, SOSP-9607-E10 and HeLa cells transfected with the novel immuno-tBid protein expression constructs. (C) SKBR-3, SOSP-9607-E10 and HeLa cells were transiently transfected with the novel immuno-tBid protein expression constructs and the suppression percentages were calculated.

counting plate, and the cell-killing ratio was determined as follows: $(\mathrm{Nc}-\mathrm{Nt}) / \mathrm{Nc} \times 100 \%$. In this formula $\mathrm{Nt}$ and $\mathrm{Nc}$ represent the number of cells in the treatment and control groups, respectively.
Flow cytometry assay and TUNEL staining for detection of apoptosis. SOSP-9607-E10 cells were seeded at a density of $1 \times 10^{5}$ cells per well on slides in 12 -well Costar transwell plates. HeLa cells stably transfected with $e 23 s F v-F d t-t B i d$ or 
A

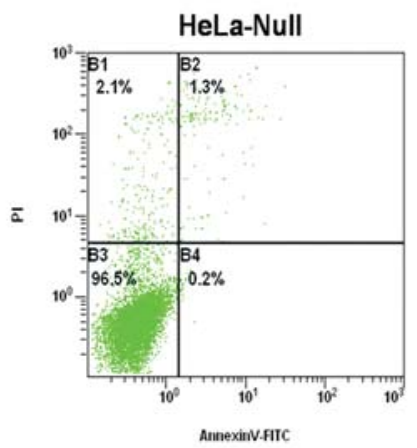

B

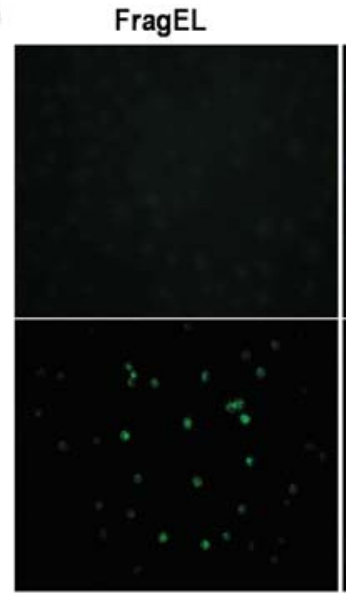

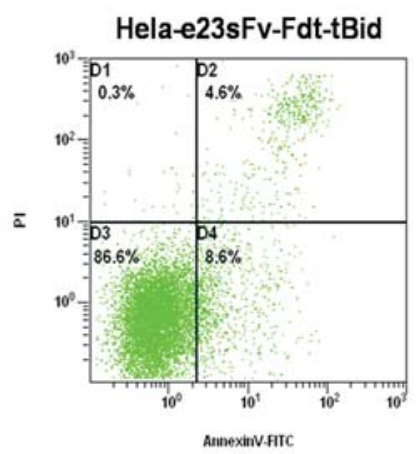

DAPI

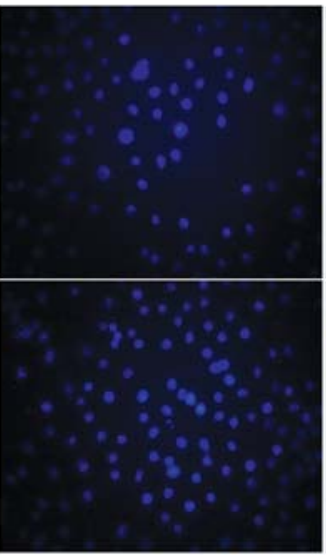

Merge

Hela-e23sFvFdt-tBid

Figure 3. SOSP-9607-E10 cells were co-cultured with immuno-tBid protein-expressing HeLa cells, and apoptosis was observed by flow cytometry (A) and TUNEL staining (B).

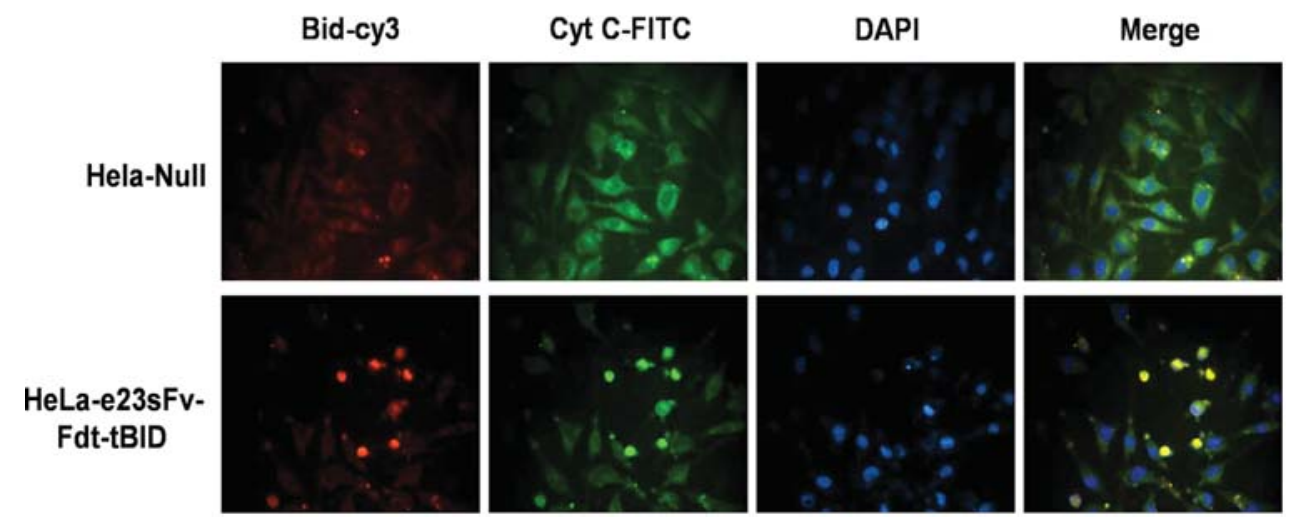

Figure 4. Secreted chimeric protein induced apoptosis through Bid and cytochrome C. SOSP-9607-E10 cells co-cultured with immuno-tBid proteinexpressing HeLa cells exhibited intensive cytoplasmic fluorescence of both Bid and cytochrome C, suggesting that apoptosis was induced by the expression of Bid and, subsequently, the secretion of cytochrome C.

control cells transfected with pCMV, were placed in the top chamber at a density of $3 \times 10^{5}$ cells per well. Cells were cocultured at E/T ratios of 3:1 for $8 \mathrm{~h}$. The apoptosis of the cells in the lower chamber was then detected by flow cytometry assay and TUNEL staining. As for flow cytometry assay, cells in the lower chamber were collected, stained with Annexin V-FITC/PI following standard procedures, and finally analyzed. As for TUNEL staining, cells were fixed, stained using the TdT-FragEL ${ }^{\mathrm{TM}}$ DNA Fragmentation
Detection kit (Calbiochem) and observed thereafter using a fluorescence microscope.

Immunofluorescence. Immunofluorescence assay was performed in order to study whether the apoptosis of the HER2-positive tumor cells was induced by tBid and subsequent release of cytochrome $\mathrm{C}$ from mitochondria. SOSP9607-E10 cells were co-cultured with HeLa-e23sFv-FdttBID or Hela-Null cells as mentioned above. After that, 
SOSP-9607-E10 cells in the lower chamber were fixed in $4 \%$ paraformaldehyde, permeabilized with PBS containing $0.1 \%$ Triton X-100, and blocked with $2 \%$ normal rabbit serum. Then the cells were stained with antibodies recognizing Bid (C20, 1:200; Santa Cruz Biotechnologies) and cytochrome C (H-104, 1:200; Santa Cruz Biotechnologies) as the primary antibodies, with biotin-linked anti-goat IgG (1:100; Santa Cruz Biotechnologies) and FITC-linked anti-rabbit IgG $(1: 100$; Sigma) as the secondary antibodies and with Cy3linked anti-biotin antibodies (1:100; Sigma) as the tertiary antibody. Nucleus staining was performed with 4,6-diamidino2-phenylindole.

Statistical analysis. Statistical analysis was performed with the SPSS 12.0 software package for Windows (SPSS). Statistical significance was defined as a value of $\mathrm{p} \leq 0.05$.

\section{Results}

Expression of HER2 in SOSP-9607-E10 cell line and clinical specimens. The HER2-positive human breast tumor cells, SKBR-3, the HER2-negative human cervical carcinoma cells, HeLa, and the subclone of a human osteosarcoma cell line with relatively high metastatic potential, SOSP-9607E10, were subjected to RT-PCR and Western blot analysis in order to determine the expression of HER 2 on both the mRNA and protein levels. As can be seen from the results of RT-PCR (Fig. 1A) and Western blot analysis (Fig. 1B), the SKBR-3 cells, in accordance with other studies, exhibited a significantly intensified expression of HER2. Though the intensity of HER2 expression in the SOSP-9607-E10 cells was not as strong as that of the SKBR-3 cells, it was, indeed, markedly stronger than that of the HeLa cells.

As for the immunohistochemical staining of the clinical specimens, 18 out of the 48 specimens (37\%) exhibited moderate or strong immunostaining of HER2. Fig. 1C shows representative images of a positively- and negatively-stained specimen, respectively.

The novel immuno-tBid can induce cell death in SOSP-9607E10 cells. As can be seen from Fig. 2A, the novel recombinant gene consisted of a signal peptide, the HER2 antibody, e $23 \mathrm{sFv}$, the furin cleavage sequence, Fdt, and tBid (bid $\Delta 1-60$ ). In order to test the possible suppressing effect of this recombinant molecule on HER2-overexpressing cells, the viability of the cells transiently transfected with the recombinant molecule, was studied by MTT assay and trypan blue staining. Apparent cell death was observed in the HER2-positive SKBR-3 and SOSP-9607-E10 cells, but not in the HER2negative HeLa cells (Fig. 2B and C).

Secreted e23sFv-Fdt-tBid protein can induce apoptosis in SOSP-9607-E10 cells. In order to investigate the proapoptotic effect of the secreted recombinant protein, HeLa cells stably transfected with the recombinant gene were cocultured with SOSP-9607-E10 cells. As can be seen from the flow cytometry results (Fig. 3A), a large percentage (16.1\%) of SOSP-9607-E10 cells underwent apoptosis after they were co-cultured with HeLa cells expressing the novel immunotBid. This pro-apoptotic effect was also confirmed by TUNEL staining (Fig. 3B). A significant number of SOSP-9607-E10 cells in the Hela-e23sFv-Fdt-tBid group exhibited green fluorescence, indicating that they are apoptotic. The above results suggest that the novel immuno-tBid secreted by transfected HeLa cells is very potent in inducing apoptosis in HER2-positive osteosarcoma cells.

Secreted fusion protein induced apoptosis through Bid and cytochrome $C$. Immunofluorescence staining was performed to further prove that the pro-apoptotic effect of the fusion protein was induced by the translocation of Bid to the cytoplasm and the consequent cytochrome $\mathrm{C}$ release from mitochondria. As shown in Fig. 4, SOSP-9607-E10 cells cocultured with HeLa cells stably secreting the recombinant protein, exhibited intensive cytoplasmic fluorescence of both $\mathrm{Bid}$ and cytochrome $\mathrm{C}$. These results suggest that, after the internalization of the recombinant protein, tBid translocates into the cytoplasm, thus inducing cythchrome $\mathrm{C}$ release and finally causing apoptosis in HER2-positive osteosarcoma cells.

\section{Discussion}

In the present study, we constructed the novel immunoproapoptotic protein, e $23 \mathrm{sFv}-\mathrm{Fdt}-\mathrm{Bid} \Delta \mathrm{1}-60$, and confirmed its efficient pro-apoptotic effect on HER2-overexpressing osteosarcoma cells.

In the past decade, we carried out a series of studies on antibody-directed and cell-mediated cancer immunotherapy by combining the specificity of antibodies and the potent cytotoxicity of pro-aoptotic proteins. Up to now, a number of pro-apoptotic effectors, including caspase-3 (19), caspase-6 (20), granzyme B (21), tBid $(16,17)$ and apoptosis inducing factor (AIF) (22), have been used to construct our immunoproapoptotic proteins and have been confirmed to be efficient in inducing targeted apoptosis both in vitro and in vivo. Among all these recombinant proteins, immuno-tBid has been proven to be a bright prospect for future therapeutic application, as Bid is an intracellular link connecting the receptor- and mitochondrial-mediated apoptotic pathways $(23,24)$ and thus guarantees efficiency when either pathway is unavailable in tumor cells. However, one obstacle that hinders the clinical application of immuno-tBid is its immunogenicity. In order to reduce the immunogenicity of immuno-tBid, we further refined it by replacing the PEA translocation domain with a smaller exogenous fragment, a furin sensitive sequence derived from the diphtheria toxin $\left(\mathrm{A}_{187} \mathrm{GNRVRRSVG}_{196}, \mathrm{Fdt}\right)$. As this new immuno-proapoptotic protein consists mainly of humanized antibodies and human apoptotic effectors, it possesses putatively minor immunogenicity. With reduced possibility of inducing immune response and systemic toxicity, this novel immuno-tBid promises improved therapeutic utility for the long-term treatment of tumors that overexpress HER2.

The transmembrane glycoprotein, HER2, classified as a member of the epidermal growth factor receptor (EGFR) family, has been reported to be overexpressed in many adenocarcinomas, including breast, ovarian, lung and stomach tumors (25-28), and is thus recognized as an ideal molecular target for cancer gene therapy. Although HER2 is generally 
recognized as an indicator of tumor migration and poor prognosis, its role in osteosarcoma is controversial (29-37). In this study, we investigated the expression of HER 2 in SOSP-9607-E10 cells, a previously established subculture of osteosarcoma cells with relatively high metastatic potential (38). In accordance with our previous flow cytometry results (17), RT-PCR and Western blot analysis in this study demonstrated an increased HER2 expression in SOSP-9607-E10 cells. Further immunohistochemical analysis of the HER2 expression in clinical specimens also revealed overexpression in $37 \%$ of all the examined specimens. Taking all these results together, we hypothesized that HER2 could be an effective target for the immunotherapy of osteosarcoma, especially the type with high metastatic potential.

In order to verify the HER2-targeted suppressing effect of the novel immuno-tBid, we transfected the recombinant gene into SOSP-9607-E10 cells, HER2-positive SKBR-3 cells, as well as into HER2-negative HeLa cells, and compared the vitality of these cells after transient transfection. The effective destruction of SOSP-9607-E10 cells by the novel immunotBid was confirmed by both MTT assay and trypan blue staining. Significant cell death was observed in both SOSP9607-E10 and SKBR-3 cells, but not in HeLa cells, at $48 \mathrm{~h}$ after transfection. The percentage of cell destruction was 39.7 and $31 \%$ for the SKBR-3 and SOSP-9607-E10 cells, respectively. These results confirm our hypothesis that the recombinant gene encoding the novel immuno-tBid can induce the targeted suppression of HER2-positive SOSP-9607-E10 cells.

As for future therapeutic application, our aim is to transfect the novel immuno-tBid gene into lymphocytes. These modified lymphocytes would secret the immunotoxin after they 'home' to the tumor site. It is believed that the secreted novel immuno-tBid would bind to HER2-overexpressing osteosarcoma cells, and subsequently induce apoptosis in these tumor cells. In this preliminary study, we transfected the recombinant gene into HeLa cells and investigated the pro-apoptotic effect of the secreted immuno-toxin on SOSP9607-E10 cells. As expected, secreted immunotoxin was detected in culture medium of transfected HeLa cells by Western blot analysis (data not shown). Moreover, TUNEL staining and flow cytometry revealed that a significant number of the SOSP-9607-E10 cells co-cultured with modified HeLa cells underwent a typical process of apoptosis. With the understanding that tBid exerts its pro-apoptotic effect by inducing the release of pro-apoptotic factors, such as cytochrome $\mathrm{C}$, AIF, and procaspase 9, from mitochondria $(22,23)$, we performed the immunofluorescence assay in order to further confirm the pro-apoptotic effect of the novel immuno-tBid on SOSP-9607-E10 cells. Again, the potent pro-apoptotic effect of the novel immuno-tBid was proven by the fluorescence of both Bid and its downstream pro-aoptotic factor, cytochrome $\mathrm{C}$, in the cytoplasm.

Thus, it can be conclude that, the novel immuno-tBid with the minimized exogenous fragment can effectively and selectively destroy HER2-positive osteosarcoma cells. This novel immunotoxin could be a promising candidate for the future development of long-term immunotherapies with maximal cell-suppressing ability and minimal non-specific damage to normal tissues.

\section{Acknowledgements}

This study was supported by grants from the National Basic Research Program of China (no. 2010CB529905), the National High Technology Research and Development Program of China (nos. 2006AA02A255 and 2007AA021104), as well as the National Natural Science Foundation of China (nos. 30901784, 30973409, 30873027, 30701006 and 30330610).

\section{References}

1. Matthey B, Engert A and Barth S: Recombinant immunotoxins for the treatment of Hodgkin's disease. Int J Mol Med 6: 509-514, 2000.

2. Schindler J, Sausville E, Messmann R, et al: The toxicity of deglycosylated ricin A chain containing immunotoxins in patients with non-Hodgkin's lymphoma is exacerbated by prior radiotherapy: a retrospective analysis of patients in five clinical trials. Clin Cancer Res 7: 255-258, 2001.

3. Kreitman RJ, Wilson WH, Bergeron K, et al: Efficacy of the anti-CD22 recombinant immunotoxin BL22 in chemotherapyresistant hairy-cell leukemia. N Engl J Med 345: 241-247, 2001.

4. Kreitman RJ: Chimeric fusion proteins-Pseudomonas exotoxinbased. Curr Opin Investig Drugs 2: 1282-1293, 2001.

5. Henry CJ, Buss MS, Hellström I, et al: Clinical evaluation of BR96 sFv-PE40 immunotoxin therapy in canine models of spontaneously occurring invasive carcinoma. Clin Cancer Res 11: 751-755, 2005.

6. Martin PJ, Pei J, Gooley T, et al: Evaluation of a CD25-specific immunotoxin for prevention of graft-versus-host disease after unrelated marrow transplantation. Biol Blood Marrow Transplant 10: 552-560, 2004.

7. Wong L, Suh DY and Frankel AE: Toxin conjugate therapy of cancer. Semin Oncol 32: 591-595, 2005.

8. Chen SY, Yang AG, Chen JD, et al: Potent antitumour activity of a new class of tumour-specific killer cells. Nature 385: 78-80, 1997.

9. Von Minckwitz G, Harder S, Hövelmann S, et al: Phase I clinical study of the recombinant antibody toxin scFv(FRP5)ETA specific for the ErbB2/HER2 receptor in patients with advanced solid malignomas. Breast Cancer Res 7: R617-R626, 2005.

10. Posey JA, Khazaeli MB, Bookman MA, et al: A phase I trial of the single-chain immunotoxin SGN-10 (BR96 sFv-PE40) in patients with advanced solid tumours. Clin Cancer Res 8: 3092-3099, 2002.

11. Hall PD, Virella G, Willoughby T, et al: Antibody response to DTGM, a novel fusion toxin consisting of a truncated diphtheria toxin (DT) linked to human granulocyte-macrophage colony stimulating factor (GM), during a phase I trial of patients with relapsed or refractory acute myeloid leukemia. Clin Immunol 100: 191-197, 2001.

12. Benbrook DM: An ELISA method for detection of human antibodies to an immunotoxin. J Pharmacol Toxicol Methods 47: 169-175, 2002.

13. Onda M, Willingham M, Wang QC, et al: Inhibition of TNFalpha produced by Kupffer cells protects against the nonspecific liver toxicity of immunotoxin anti-Tac(Fv)-PE38, LMB-2. J Immunol 165: 7150-7156, 2000.

14. Baluna R and Vitetta ES: Vascular leak syndrome: a side effect of immunotherapy. Immunopharmacology 37: 117-132, 1997.

15. Erickson HA, Jund MD and Pennell CA: Cytotoxicity of human RNase-based immunotoxins requires cytosolic access and resistance to ribonuclease inhibition. Protein Eng Des Sel 19: 37-45, 2006.

16. Qiu XC, Xu YM, Wang F, et al: Single-chain antibody/ activated BID chimeric protein effectively suppresses HER2positive tumor growth. Mol Cancer Ther 7: 1890-1899, 2008

17. Shan LQ, Qiu XC, Xu YM, et al: scFv-mediated delivery of truncated BID suppresses HER2-positive osteosarcoma growth and metastasis. Cancer Biol Ther 11: 1717-1722, 2008.

18. Wang T, Zhao J, Ren JL, et al: Recombinant immunoproapoptotic proteins with Furin site can translocate and kill HER2positive cancer cells. Cancer Res 67: 11830-11839, 2007.

19. Jia LT, Zhang LH, Yu CJ, et al: Specific tumouricidal activity of a secreted pro-apoptotic protein consisting of HER2 antibody and constitutively active caspase-3. Cancer Res 63: 3257-3262, 2003. 
20. Xu YM, Wang LF, Jia LT, et al: A caspase-6 and antihuman epidermal growth factor receptor-2 (HER2) antibody chimeric molecule suppresses the growth of HER2-overexpressing tumours. J Immunol 173: 61-67, 2004.

21. Zhao J, Zhang LH, Jia LT, et al: Secreted antibody/granzyme B fusion protein stimulates selective killing of HER2overexpressing tumour cells. J Biol Chem 279: 21343-21348, 2004.

22. Yu CJ, Jia LT, Meng YL, et al: Selective pro-apoptotic activity of a secreted recombinant antibody/AIF fusion protein in carcinomas overexpressing HER2. Gene Ther 13: 313-320, 2006.

23. Li H, Zhu H, Xu CJ, et al: Cleavage of BID by caspase 8 mediates the mitochondrial damage in the Fas pathway of apoptosis. Cell 94: 491-501, 1998.

24. Luo X, Budlhardjo I, Zou H, et al: Bid, a Bcl-2 interacting protein, mediates cytochrome $\mathrm{c}$ release from mitochondria in response to activation of cell surface death receptors. Cell 94: 481-490, 1998.

25. Schneider PM, Praeuer HW, Stoeltzing O, et al: Multiple molecular marker testing (p53, C-Ki-ras, c-erbB-2) improves estimation of prognosis in potentially curative resected nonsmall cell lung cancer. Br J Cancer 83: 473-479, 2000.

26. Rasmuson T, Grankvist K and Ljungberg B: Soluble ectodomain of c-erbB-2 oncoprotein in relation to tumour stage and grade in human renal cell carcinoma. Br J Cancer 75: 1674-1677, 1997.

27. Slamon DJ, Godolphin W, Jones LA, et al: Studies of the HER-2/ neu proto-oncogene in human breast and ovarian cancer. Science 244: 707-712, 1989.

28. Menard S, Fortis S, Castiglioni F, et al: HER2 as a prognostic factor in breast cancer. Oncology 61: 67-72, 2001.

29. Gorlick R, Huvos AG, Heller G, et al: Expression of HER2/ERBB2 correlates with survival in osteosarcoma. J Clin Oncol 17: 2781-2788, 1999.
30. Onda M, Matsuda S, Higaki S, et al: ErbB2 expression is correlated with poor prognosis for patients with osteosarcoma. Cancer 77: 71-78, 1996.

31. Ferrari S, Bertoni F, Zanella L, et al: Evaluation of P-glycoprotein, HER-2/ErbB2, p53, and Bcl-2 in primary tumor and metachronous lung metastases in patients with high-grade osteosarcoma. Cancer 100: 1936-1942, 2004.

32. Anninga JK, van de Vijver MJ, Cleton-Jansen AM, et al: Overexpression of the HER-2 oncogene does not play a role in high-grade osteosarcomas. Eur J Cancer 40: 963-970, 2004

33. Fellenberg J, Krauthoff A, Pollandt K, et al: Evaluation of the predictive value of Her-2/neu gene expression on osteosarcoma therapy in lasermicrodissected paraffin-embedded tissue. Lab Invest 84: 113-121, 2004.

34. Zhou H, Randall RL, Brothman AR, et al: Her-2/neu expression in osteosarcoma increases risk of lung metastasis and can be associated with gene amplification. J Pediatr Hematol Oncol 25: 27-32, 2003.

35. Thomas DG, Giordano TJ, Sanders D, et al: Absence of HER 2/neu gene expression in osteosarcoma and skeletal Ewing's sarcoma. Clin Cancer Res 8: 788-793, 2002.

36. Akatsuka T, Wada T, Kokai Y, et al: ErbB2 expression is correlated with increased survival of patients with osteosarcoma. Cancer 94: 1397-1404, 2002.

37. Maitra A, Wanzer D, Weinberg AG, et al: Amplification of the HER-2/neu oncogene is uncommon in pediatric osteosarcomas. Cancer 92: 677-683, 2001.

38. Chen X, Yang TT, Wang W, et al: Establishment and characterization of human osteosarcoma cell lines with different pulmonary metastatic potentials. Cytotechnology 61: 37-44, 2009. 